\title{
Two-stage liver transplantation in a surgically complicated liver failure patient after hepatic tumor resection
} -A case report-

\author{
Sang Hyun Lee, Soo Hyun Yang, and Gaab Soo Kim \\ Department of Anesthesiology and Pain Medicine, Samsung Seoul Hospital, Samsung Medical Center, Sungkyunkwan University \\ School of Medicine, Seoul, Korea
}

Two-stage liver transplantation, involving a total hepatectomy with a temporary portocaval shunt followed by liver transplantation, requires intensive perioperative care, especially during the prolonged anhepatic period. The pathophysiology and management of this prolonged anhepatic state is not fully elucidated and the proper management during this period is a great challenge to clinicians in the intensive care unit and anesthesiologists. We report a case and management of a total hepatectomy with a temporary portocaval shunt followed by living-donor liver transplantation in a patient with a surgically complicated liver failure after a hepatic tumor resection. (Korean J Anesthesiol 2010; 59: 348-352)

Key Words: Anhepatic, Hepatectomy, Liver failure, Liver transplantation, Portocaval shunt, Two-stage procedure.

Two-stage liver transplantation (LT), involving a total hepatectomy with a temporary portocaval shunt followed by LT, requires intensive perioperative care, especially during the prolonged anhepatic period. Since its first introduction by Ringe et al. [1] in the late 1980s, this procedure has been investigated and performed to stabilize the hemodynamic and metabolic state of patients in acute hepatic or primary graft failure awaiting a liver graft [2-5]. However, a prolonged anhepatic state is still a challenge to clinicians in the intensive care units and anesthesiologists because the pathophysiological changes and proper management of the anhepatic state are not completely understood [6]. The clinical status of an individual patient at the time of a total hepatectomy and proper care of the anhepatic state may affect the outcome of the patient after this two-stage procedure [2]. We report a case and perioperative care of a total hepatectomy with a temporary portocaval shunt followed by living-donor LT in a patient with surgically complicated liver failure after a hepatic tumor resection.

\section{Case Report}

A 42-year-old woman (weight: $48.8 \mathrm{~kg}$, height: $159 \mathrm{~cm}$ ), who underwent an extensive tumor resection for Klatskin tumor, Bismuth type IV, developed acute hepatic failure in

Received: February 2, 2010. Revised: March 2, 2010. Accepted: March 17, 2010.

Corresponding author: Gaab Soo Kim, M.D., Ph.D., Department of Anesthesiology and Pain Medicine, Samsung Seoul Hospital, Samsung Medical Center, Sungkyunkwan University School of Medicine, 50, Irwon-dong, Gangnam-gu, Seoul 135-710, Korea. Tel: 82-2-3410-0360, Fax: 82-2-3410-0361, E-mail: gskim@skku.edu

ㄷ) This is an open-access article distributed under the terms of the Creative Commons Attribution Non-Commercial License (http:// creativecommons.org/licenses/by-nc/3.0/), which permits unrestricted non-commercial use, distribution, and reproduction in any medium, provided the original work is properly cited. 
the immediate postoperative period and was listed for an emergency LT.

A preoperative laboratory and radiology evaluation before the tumor resection were within the normal limits except for an elevated liver function profile (aspartate aminotransferase 170 $\mathrm{U} / \mathrm{L}$, alanine aminotransferase $100 \mathrm{U} / \mathrm{L}$ ) due to the underlying Klatskin tumor. The bleeding diatheses at the intensive care unit (ICU) were as follows: hemoglobin and platelet count decreased from $10.4 \mathrm{~g} / \mathrm{dl}$ and $103 \times 10^{3} / \mu \mathrm{l}$ to $4.8 \mathrm{~g} / \mathrm{dl}$ and $60 \times$ $10^{3} / \mu \mathrm{l}$, respectively, within the first hour at the ICU. Prothrombin time (PT) expressed in the international normal range (INR), activated partial thrombin time (aPTT), fibrinogen, fibrin degradation product, D-dimer, antithrombin III activity, plasminogen activity, protein $\mathrm{C}$ activity were 2.03 (normal [nl], $0.9-1.1), 104.5$ seconds (nl, 29-42 seconds), $97 \mathrm{mg} / \mathrm{dl}$ (nl, $180-380 \mathrm{mg} / \mathrm{dl}$ ), $>5 \mu \mathrm{g} / \mathrm{ml}$ (nl, $<5 \mu \mathrm{g} / \mathrm{ml}), 3.23 \mu \mathrm{g} / \mathrm{ml}(\mathrm{nl}, 0-$ $0.5 \mu \mathrm{g} / \mathrm{ml}$ ), 41\% (nl, 83-123\%), 30\% (nl, 75-112\%), 43\% (nl, 80-161\%), respectively. Metabolic acidosis of $\mathrm{pH}<7.15$ with a progressively deteriorating base deficit $>15 \mathrm{mmol} / \mathrm{L}$ and exacerbating hypocalcemia $<0.8 \mathrm{~mol} / \mathrm{L}$ and hyperglycemia $>200$ $\mathrm{mg} / \mathrm{dl}$ were observed throughout the ICU stay. Continuous infusions of calcium gluconate at $1 \mathrm{mg} / \mathrm{kg} / \mathrm{hr}$ and $5 \%$ albumin were given. Bicarbonate and insulin were given intermittently at the discretion of the clinician. At the ICU, exsanguinating bleeding of more than $1,000 \mathrm{ml} / \mathrm{hr}$ was seen through a drainage immediately after the tumor resection. During the 11 hour stay at the ICU before receiving a total hepatectomy, more than 25 units of packed red blood cells, 16 units of each fresh frozen plasma, cryoprecipitate and platelet concentrates were administered along with a continuous infusion of $10 \mu \mathrm{g} / \mathrm{kg} /$ min dopamine, $0.35 \mu \mathrm{g} / \mathrm{kg} / \mathrm{min}$ norepinephrine to maintain the systolic blood pressure between 50 and $80 \mathrm{mmHg}$, and the diastolic blood pressure between 30 and $65 \mathrm{mmHg}$. The urine output was maintained between 20 and $100 \mathrm{ml} / \mathrm{hr}$ during the ICU stay. The patient was indicated to undergo a total hepatectomy with a temporary portocaval shunt while awaiting a liver graft due to the uncontrollable bleeding and hepatic toxic syndrome, which was postulated to be caused by acute hepatic necrosis and the resultant coagulation abnormality. During the two hour and 40 minute surgical procedure, crystalloid 8,100 $\mathrm{ml}$, packed red blood cells eight units, fresh frozen plasma four units, platelet concentrates eight units and cryoprecipitate six units were administered to replace the estimated blood loss of 4,500 $\mathrm{ml}$. The total urine output was $330 \mathrm{ml}$.

After the total hepatectomy and portocaval shunt, the patient was placed at the ICU and medical care for the anhepatic state was provided. During the anhepatic period, an hourly monitoring of the arterial blood gas analysis with an intermittent the ionic calcium level and dieresis were the primary concern of the ICU management. Arterial blood gas analyses showed an alleviation of the metabolic acidosis from arterial $\mathrm{pH}<7.15$ and base deficit $>15 \mathrm{mmol} / \mathrm{L}$ during the acute liver failure status to $\mathrm{pH} 7.15-7.25$ and base deficit of 10$15 \mathrm{mmol} / \mathrm{L}$ during the anhepatic period. Metabolic acidosis was accompanied by slight hyperchloremia ranging 110-115 $\mathrm{mmol} / \mathrm{L}$ (nl, <108 mmol/L). However, the potassium level was within the normal range of $3.5-3.8 \mathrm{mmol} / \mathrm{L}$ throughout the ICU stay before the hepatectomy. The magnesium level decreased only slightly ranging $1.6-1.7 \mathrm{mg} / \mathrm{dl}$ and the sodium level was maintained between 144 and $146 \mathrm{mmol} / \mathrm{L}$. Persistent ionic hypocalcemia $<0.8 \mathrm{mmol} / \mathrm{L}$ was observed despite the continuous infusion of calcium gluconate supplementation at $1 \mathrm{mg} / \mathrm{kg} / \mathrm{hr}$. Other continuous supplementations including sodium bicarbonate, magnesium, $5 \%$ albumin, $20 \%$ mannitol and dextrose solution were provided as needed. There was no rapid correction of hyponatremia or an increase in the sodium level owing to the use of sodium bicarbonate in this case. There were no neurological deficits following the abnormal changes in sodium level or signs of hepatic encephalopathy, even though comprehensive neurological examinations were not feasible due to the patient's sedated and intubated condition. The serum glucose level was maintained between 100 and 200 $\mathrm{mg} / \mathrm{dl}$. A warm blanket was applied to maintain the normal body temperature. An inotropic support with a continuous infusion of $5 \mu \mathrm{g} / \mathrm{kg} / \mathrm{min}$ dopamine maintained the systolic blood pressure between 80 and $100 \mathrm{mmHg}$ and diastolic blood pressure between 60 and $80 \mathrm{mmHg}$. At the discretion of the ICU specialist, 12 units of packed red blood cells, 10 units of fresh frozen plasma, 16 units of platelet concentrates and 14 units of cryoprecipitate were given over an 11-hour period at the ICU before the liver transplant. An elevated creatinine level of $1.58 \mathrm{mg} / \mathrm{dl}$ and urine out of $<10 \mathrm{ml} / \mathrm{hr}$ were observed, but continuous renal replacement therapy (CRRT) was not applied. The coagulation profile during the anhepatic period showed a prolongation of PT (INR) around 2, aPTT around $100 \mathrm{~s}$ and fibrinogen $<100 \mathrm{mg} / \mathrm{dl}$. Cytokine or other toxin related laboratory analyses were not performed in this patient. No artificial liver support, such as molecular adsorbent recirculating system (MARS), was applied in this patient because she was not considered a suitable candidate.

The patient was anhepatic for 15 hours before reperfusion of the liver in the living donor liver transplantation. Anesthesia was induced and maintained with desflurane at an oxygen fraction of 0.5 under routine monitoring with electrocardiography, $\mathrm{SpO}_{2}$, radial and femoral arterial pressure, pulmonary arterial pressure and cardiac output. Continuous vasoactive support with $5 \mu \mathrm{g} /$ $\mathrm{kg} / \mathrm{min}$ dopamine and $0.1-0.2 \mu \mathrm{g} / \mathrm{kg} / \mathrm{min}$ norepinephrine were maintained. The hemodynamic profile was stable throughout the operation before and after reperfusion of the transplanted liver. During the seven hour and 40 minute LT operation, 7,500 
$\mathrm{ml}$ crystalloid, $500 \mathrm{ml}$ colloid, $650 \mathrm{ml}$ albumin, eight units of packed red blood cell, four units of fresh frozen plasma, nine units of cryoprecipiate and one unit of platelet apheresis were administered.

Postoperative care at the ICU involved cardiovascular and respiratory support with a CCRT. On the ninth day after LT, the patient became alert and on the 16th day she was extubated for one week until reintubated due to pulmonary effusion related respiratory failure. The acute renal insufficiency with urine output of $<5 \mathrm{ml} / \mathrm{hr}$ and creatinine level between 1.5 and $2.0 \mathrm{mg} / \mathrm{dl}$ persisted despite the best efforts to improve the renal function. The patient also suffered peritonitis from bowel anastomosis leakage and underwent two additional operations for anastomosis site repair. The sustained septic conditions throughout the ICU stay aggravated and progressed to multiorgan failure. The patient died on postoperative day 65 .

A

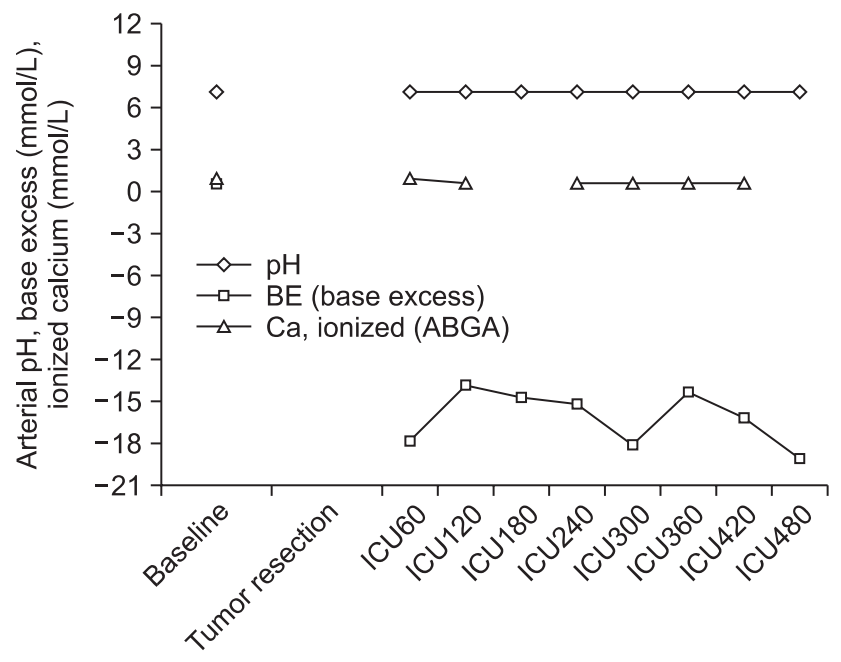

C

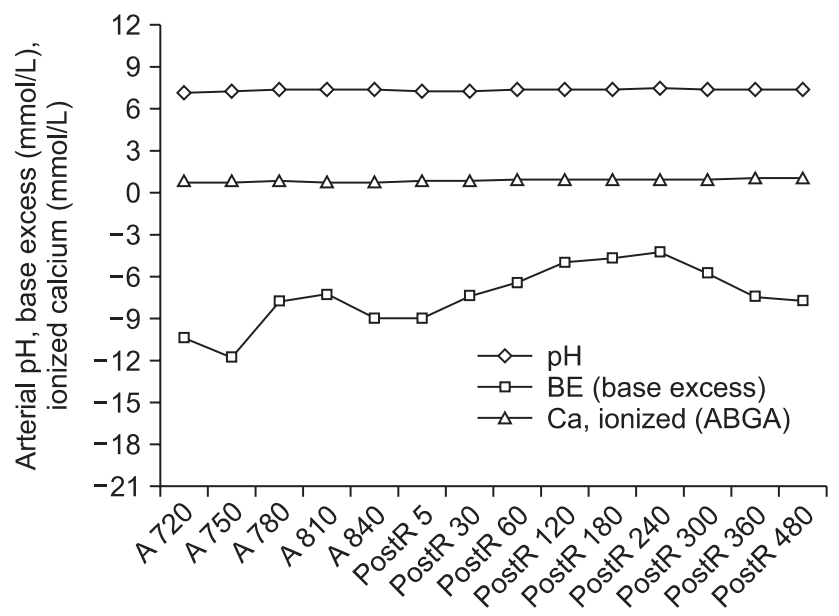

\section{Discussion}

A total hepatectomy with a portocaval shunt was performed as a bridging procedure to a LT because the patient developed acute liver failure, which manifested as uncontrollable massive bleeding and so-called "toxic liver syndrome" immediately after a surgical resection of a Klatskin tumor.

Toxic liver syndrome is one of the major indications for this procedure and it is known to occur after acute or fulminant hepatic necrosis that is often complicated by multiorgan failure, such as cardiovascular shock, renal failure, pulmonary insufficiency and cerebral dysfunction. The multiorgan involvement is caused by many factors including toxic metabolites released from the necrotized liver and endogenous vasoactive materials. The mortality of this condition is rated nearly $100 \%$. Therefore, the elimination of the necrotized liver was
B

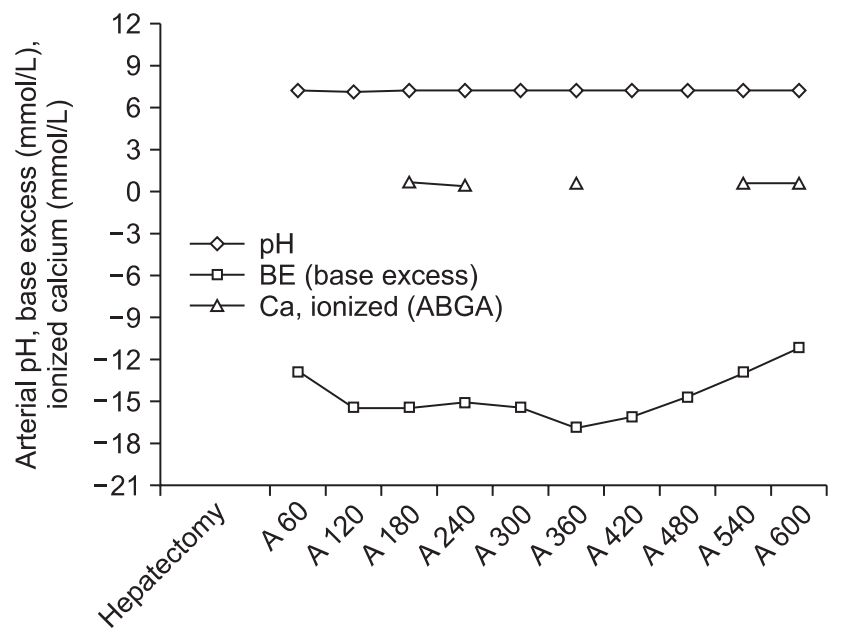

Fig. 1. Changes in the arterial $\mathrm{pH}$, base excess and ionized calcium level at the baseline, during the intensive care unit (ICU) stay before total hepatectomy, during the anhepatic state (A), and after reperfusion of the liver transplant (postR). (A) Arterial pH, base excess and ionized calcium level at the baseline and during the initial ICU stay after tumor resection. (B) Arterial $\mathrm{pH}$, base excess and ionized calcium level during the anhepatic ICU stay period after the total hepatectomy. (C) Arterial $\mathrm{pH}$, base excess and ionized calcium level during the anhepatic liver transplantation procedure and after reperfusion. The base excess and ionized calcium level vary within the time period of the ICU care before the total hepatectomy and during the anhepatic period with a continuous infusions of sodium bicarbonate and calcium gluconate (not indicated in the figure). However, these figures delineate the persistent low BE and calcium level despite the continuous supplementation during the anhepatic period until reperfusion of the liver transplant. The numbers in the $\mathrm{x}$-axis indicate the time in minutes. 
justified in this concept and several clinical reports showed that a total hepatectomy and portocaval shunt stabilized the hemodynamic and metabolic state, and improved the portal venous drainage and decompression. Our patient also became hemodynamically stable with fewer requirements of blood transfusion and inotropic support, and metabolic acidosis progression was slowed. Her arterial blood gas analysis with the ionic calcium level improved after the total hepatectomy and portocaval shunt (Fig. 1). Our patient did not develop liver failure-related neurological dysfunction during the ICU stay and monitoring of the ICP was not required. However patients with acute or fulminant liver failure, especially in cases of acute-onchronic liver diseases, should be considered for ICP monitoring $[7,8]$. Monitoring of ICP is not without complications, such as intracranial hemorrhage and infection [9]. However, since an elevated ICP is one of the major cases of mortality before a liver transplant, its beneficial role in managing ICP should be weighed against its complications. Although the consequences of a prolonged anhepatic state are not fully established, adequate management during the anhepatic period is essential to the survival until a graft is ready. Previous reports stated that patients in an anhepatic state rapidly develop hypoglycemia, hypothermia, oliguria, renal failure and hypocalcemia $[6,10]$.

Anhepatic patients develop hypoglycemia because the liver is the main source of glycogen storage, therefore needs to be replaced with an intravenous glucose infusion. Hypothermia needs to be managed with a warm blanket and the body temperature needs to be maintained. Oliguria and renal failure often develop rapidly within 24 hours of an anhepatic state, and early renal dysfunction might indicate a poor prognosis. Renal insufficiency is reported to be related to the prevalence of postoperative sepsis. Supports to provide renal perfusion by diuretics, mannitol or renal dose dopamine infusion may be considered [8]. Some clinicians run CCRT and some even prefer to apply it prophylactically. Our patient also became rapidly oliguric but CCRT was not applied. Ionic hypocalcemia is severe in prolonged anhepatic periods because citrate from the blood product accumulates without being metabolized due to the absence of liver [11]. Our patient also showed persistent hypocalcemia despite the continuous infusion of calcium supplements throughout the anhepatic period (Fig. 1). Vigorous calcium replacement is recommended because severe hypocalcemia can result in negative cardiac output and hemodynamic compromise. Coagulation defects in anhepatic patients are not as severe as indicated by the conventional laboratory results, and may be treated with fresh frozen plasma and fibrinogen [8]. Anhepatic patients also need to be cautioned regarding sepsis and multiorgan failure as these are reportedly the main cause of mortality. Although our patient also suffered and died of sepsis and multiorgan failure, her septic condition was confounded by peritonitis that was complicated from bowel anastomosis leakage. It is unclear if the complicated peritonitis persisted because of the patient's immune downfall. The transplanted liver was considered to be in good function as the patient recovered consciousness on the ninth postoperative day without any signs of graft rejection. Therefore, the patient would have survived the liver transplant and lived had it not been for the complicated bowel anastomosis leakage.

The safety margin of time for a prolonged anhepatic state is also not known. The maximum duration of 41 hours, 60 hours and a range of 6.58 to 72.5 hours were reported by Ringe et al. [2], Bentdal et al. [4], and Oldhafer et al. [12] respectively. In this patient, the anhepatic time was 15 hours. The survival rate varied from study to study, but was $>60 \%$ overall [10]. The permissible anhepatic time for a better prognosis or its correlation with a deterioration of the patient's condition are difficult to define because the survival varies according to the clinical status of the patient at the time of the total hepatectomy and the effectiveness of supportive care before and after the two-stage procedure [2]. The best prognosis appears to depend mostly on the patient's factors and prompt availability of a liver graft. In this regard, a living donor liver transplant may be advocated even in an emergent situation because a cadaveric donor may not be available in a timely manner.

Along with this two-stage procedure, there have been other efforts, such as the use of liver support devices, to sustain the acute or fulminant liver failure patients until a liver graft is ready $[13,14]$. Bioartificial liver support using charcoal columns and extracorporeal devices filled with the liver tissue has been reported [13], and more recently the MARS has been introduced as a nonbiological liver support device $[14,15]$. The application of these devices is a relatively common clinical practice because they remove toxic materials from the patients' plasma, thus providing the patients with more time to wait for a liver graft by improving the hemodynamic profiles and alleviating the increased intracranial pressure. The MARS was not applied in this patient after the extensive hepatic tumor resection because she suffered from acute liver failure not confounded by chronic liver diseases and was not considered a suitable candidate. Her medical condition did not progress to develop hepatic encephalopathy or other extensive toxic status that would necessitate the application of the MARS during the anhepatic period.

Different anesthetic demands and management schemes are needed for the LT in a two stage procedure compared to LT in general. In the two stage procedure, LT is not prone to develop massive bleeding as is the case in a routine LT procedure becuase hepatectomy and a portocaval shunt have already been performed. In addition, the hemodynamic instability originating from clamping of the portal vein does 
not occur during LT in the two stage procedure. Patient care at the ICU during the anhepatic period and anesthetic patient management at the beginning of the LT surgical procedure may share a uniform rationale regarding the ongoing prolonged status of the anhepatic stage even from the start of the operation compared to a routine LT. An evaluation of the calcium level and its replacement are needed more frequently at the ICU because hypocalcemia is particularly severe in these transplant recipients.

In conclusion, a total hepatectomy and temporary portocaval shunt may be performed successfully as a bridging procedure to liver transplantation if backed up with an adequate management during the anhepatic period.

\section{References}

1. Ringe B, Pichlmayr R, Lübbe N, Bornscheuer A, Kuse E. Total hepatectomy as temporary approach to acute hepatic or primary graft failure. Transplant Proc 1988; 20(1 Suppl 1): 552-7.

2. Ringe B, Lübbe N, Kuse E, Frei U, Pichlmayr R. Total hepatectomy and liver transplantation as two-stage procedure. Ann Surg 1993; 218: 3-9.

3. Henderson A, Webb I, Lynch S, Kerlin P, Strong R. Total hepatectomy and liver transplantation as a two-stage procedure in fulminant hepatic failure. Med J Aust 1994; 161: 318-9.

4. Bentdal O, Brekke IB, Olausson M, Foss A, Husberg B, Ostensen $A$, et al. Living related liver retransplantation in a 6-month-old child after 60 hours of anhepatic phase following hepatectomy of thrombosed primary liver graft. Transplant Proc 2001; 33: 2497-8.

5. Ferraz-Neto BH, Moraes-Junior JM, Hidalgo R, Zurstrassen MP, Lima IK, Novais HS, et al. Total hepatectomy and liver transplantation as a two-stage procedure for toxic liver: case reports. Transplant Proc 2008; 40: 814-6.
6. Detry O, De Roover A, Delwaide J, Hans MF, Canivet JL, Meurisse $\mathrm{M}$, et al. Prolonged anhepatic state after early liver graft removal. Hepatogastroenterology 2007; 54: 2109-12.

7. Trotter JF. Practical management of acute liver failure in the Intensive Care Unit. Curr Opin Crit Care 2009; 15: 163-7.

8. Ozier Y, Klinck JR. Anesthetic management of hepatic transplantation. Curr Opin Anaesthesiol 2008; 21: 391-400.

9. Vaquero J, Fontana RJ, Larson AM, Bass NM, Davern TJ, Shakil AO, et al. Complications and use of intracranial pressure monitoring in patients with acute liver failure and severe encephalopathy. Liver Transpl 2005; 11: 1581-9.

10. Bustamante M, Castroagudín JF, Gonzalez-Quintela A, Martinez J, Segade FR, Fernandez A, et al. Intensive care during prolonged anhepatic state after total hepatectomy and porto-caval shunt (twostage procedure) in surgical complications of liver transplantation. Hepatogastroenterology 2000; 47: 1343-6.

11. Martin TJ, Kang Y, Robertson KM, Virji MA, Marquez JM. Ionization and hemodynamic effects of calcium chloride and calcium gluconate in the absence of hepatic function. Anesthesiology 1990; 73: $62-5$.

12. Oldhafer KJ, Bornscheuer A, Frühauf NR, Frerker MK, Schlitt HJ, Ringe B, et al. Rescue hepatectomy for initial graft non-function after liver transplantation. Transplantation 1999; 67: 1024-8.

13. Hui T, Rozga J, Demetriou AA. Bioartificial liver support. J Hepatobiliary Pancreat Surg 2001; 8: 1-15.

14. Pugliese F, Ruberto F, Perrella SM, Cappannoli A, Bruno K, Martelli $\mathrm{S}$, et al. Modifications of intracranial pressure after molecular adsorbent recirculating system treatment in patients with acute liver failure: case reports. Transplant Proc 2007; 39: 2042-4.

15. Pugliese F, Novelli G, Poli L, Levi Sandri GB, Di Folco G, Ferretti $S$, et al. Hemodynamic improvement as an additional parameter to evaluate the safety and tolerability of the molecular adsorbent recirculating system in liver failure patients. Transplant Proc 2008; 40: 1925-8. 